\title{
openheart Real-world characteristics and readmissions among patients undergoing ablation for ventricular tachycardia: a retrospective database analysis of commercially insured patients in the USA
}

\author{
Vinay Mehta, ${ }^{1}$ Lee Ming Boo, ${ }^{2}$ Nader Ghaly, ${ }^{2}$ Iftekhar Kalsekar, ${ }^{3}$ Shumin Zhang, ${ }^{3}$ \\ Sashi Yadalam, ${ }^{3}$ Rahul Khanna, ${ }^{3}$ Motiur Rahman (i) ${ }^{3}$
}

\section{- Additional material is published online only. To view, please visit the journal online (http://dx.doi.org/10.1136/ openhrt-2020-001247). \\ To cite: Mehta V, Boo LM, Ghaly N, et al. Real-world characteristics and readmissions among patients undergoing ablation for ventricular tachycardia: a retrospective database analysis of commercially insured patients in the USA. Open Heart 2020;7:e001247. doi:10.1136/ openhrt-2020-001247}

Received 13 January 2020 Revised 10 July 2020 Accepted 19 August 2020

\section{Check for updates}

(C) Author(s) (or their employer(s)) 2020. Re-use permitted under CC BY-NC. No commercial re-use. See rights and permissions. Published by BMJ.

${ }^{1}$ Cardiac Electrophysiology, Aurora BayCare Medical Center, Green Bay, Wisconsin, USA ${ }^{2}$ Biosense Webster Inc, Irvine, California, USA

${ }^{3}$ Medical Device Epidemiology and Real-World Data Sciences Johnson \& Johnson, New Brunswick, New Jersey, USA

Correspondence to Dr Rahul Khanna; rkhann14@ ITS.JNJ.com

\section{ABSTRACT}

Background Radiofrequency catheter ablation is an effective treatment to alleviate symptoms and reduce recurrent implantable cardioverter-defibrillator (ICD/CRT-D) shocks in patients with ventricular tachycardia (VT). Objective To assess the characteristics and outcomes (complications, inpatient readmissions) of commercially insured patients in the USA undergoing ablation for ischaemic or non-ischaemic VT.

Methods Patients aged 18-64 years with a primary diagnosis of VT who underwent ablation between 2006 and 2015 were identified using the IBM MarketScan Commercial Database. The rate of complications including vascular complications, pericarditis, pulmonary embolism and pericardial tamponade over a 30-day post-ablation period (including index admission) was examined. Inpatient readmissions (VT-related, heart failure (HF)-related and non-VT arrhythmia-related) over the 12-month postablation period were examined. A Cox regression model was used to determine factors associated with inpatient readmissions.

Results 5242 patients (488 with ischaemic and 4754 with non-ischaemic VT) met the study criteria. The majority of VT ablations occurred in an outpatient setting ( $57 \%$ for ischaemic and $66 \%$ for non-ischaemic VT). Among complications, vascular complications were most frequent (2.05\% among ischaemic and $1.6 \%$ among non-ischaemic VT patients) over the 30-day post-ablation period. Among ischaemic VT patients, 17\%, 7.6\% and $4.7 \%$ had VTrelated, HF-related and non-VT arrhythmia-related inpatient readmissions, respectively in the 12-month post-ablation period. For non-ischaemic VT patients, these numbers were $7.5 \%, 1.7 \%$ and $3.1 \%$, respectively. Inpatient setting (vs outpatient), baseline ICD/CRT-D implantation, HF comorbidity and $\geq 2$ prior hospitalisations were associated with a higher risk of post-ablation VT-related inpatient readmissions among ischaemic VT patients. Similar factors also were associated with a higher risk of post-ablation VT-related inpatient readmission among non-ischaemic VT patients. Conclusion Setting of ablation and comorbidity status were found to influence readmission rates. Complication

\section{Key questions}

What is already known about this subject?

- Multicentre randomised controlled trials demonstrated that catheter ablation is an effective treatment option for ventricular tachycardia (VT) in lowering implantable cardioverter-defibrillator shocks and VT episodes.

What does this study add?

- Although clinical trials and observational studies have provided evidence in favour of ablation in terms of VT outcomes, most of the studies have primarily focused on patients with ischaemic VT. This is one of the first studies to examine outcomes among both ischaemic and non-ischaemic VT patients treated with catheter ablation in a real-world setting.

- Relatively low rates of complications reflect the favourable safety profile of VT ablation among ischaemic and non-ischaemic VT patients.

How might this impact on clinical practice?

- This study provides evidence in a real-world scenario that ablation can be an effective and relatively safe treatment option among patients with both ischaemic and non-ischaemic VT.

and readmission rates following VT ablation were low indicating towards the favourable safety profile of VT ablation

\section{INTRODUCTION}

Sudden cardiac death (SCD) accounts for 25\% of the 17 million cardiovascular disease-related deaths annually worldwide. ${ }^{1}$ Ventricular tachycardia (VT) degenerating to ventricular fibrillation (VF) is a frequent cause of SCD. ${ }^{2}$ Management of VT is a multipronged approach which includes treatments with antiarrhythmic drugs 
(AADs), implantable cardioverter-defibrillator (ICD/ CRT-D) and catheter ablation. ${ }^{3}$ Use of AADs in combination with/without ICDs is currently considered as the standard of care for the management of VT, with some limitations. ${ }^{34}$ AADs have not demonstrated effectiveness as primary management for VT, with the exception of beta-blockers. ${ }^{56}$ Additionally, AADs have limited use in the prevention of recurrent VT due to their toxicity and poor efficacy. ${ }^{4}$ Although ICD is considered an effective option in terminating VT episodes, and is associated with mortality reduction and improved survival among high-risk patients with left ventricular (LV) dysfunction, the painful shocks can adversely impact patient's quality of life. ${ }^{5}$ Catheter ablation is an effective treatment option for VT, and has been shown in randomised multicentre trials to reduce subsequent ICD shocks and VT episodes, as well as prevent recurrences at 6 months among patients with ischaemic heart disease. ${ }^{6} 7$ Furthermore, ablation has been found to be significantly better in prolonging time to first recurrence of any VT or VF as prophylactic therapy prior to ICD placement than ICD only. ${ }^{89}$

Although clinical trials and observational studies have provided evidence in favour of ablation in terms of VT outcomes, most of the studies have primarily focused on patients with ischaemic VT, with relatively little information available on outcomes associated with ablation among patients with non-ischaemic VT. ${ }^{31011}$ The primary objective of this study was to examine the characteristics and outcomes including complications and inpatient readmissions among commercially insured patients in the USA undergoing ablation for ischaemic or non-ischaemic VT.

\section{METHODS}

\section{Data source}

A retrospective cohort study was conducted using the IBM MarketScan Commercial Database, which is a nationally representative employer-sponsored private health insurance database with de-identified patient level information and in compliance with the Health Information Portability and Accountability Act of $1996 .{ }^{10}$ This database contains medical and prescription drug insurance claims for more than 138 million individuals in the USA and includes information on inpatient admissions, outpatient services, prescription drugs and enrolment associated with the provision of healthcare services.

\section{Study population}

The study cohort included patients aged 18-64 years with a primary diagnosis of VT (International Classification of Diseases, 9th revision and 10th revision, Clinical Modification (ICD-9/ICD-10) diagnostic codes 427.1, I47.2) who underwent catheter ablation (ICD-9/ICD-10 codes: 37.34 , 02583ZZ; current procedural terminology codes: 93652, 93654) in an inpatient or outpatient setting between 1 January 2006 and 31 December 2015. The date of first ablation during this time period was considered as 'index ablation'. Patients were required to be continuously enrolled in the insurance plan for at least 6 months in the pre-index ablation period. No continuous enrolment was applied in the post-index period and patients were followed until they lose enrolment or reach the end of the follow-up period (1 year). Patients were excluded if they had a primary or secondary procedure of catheter ablation with a primary or secondary diagnosis of VT or had undergone valvular procedures during the 6 -month pre-index period, had a ventriculotomy or atriotomy procedure performed in the 2-month pre-index period, and had systemic infection in the 2-week pre-index period. Patients were stratified across ischaemic and non-ischaemic VT based on the presence or absence of myocardial infarction (MI) (ICD-9/ICD-10 codes: 412, 410, I21, I22 and I25.2), respectively in the 6-month pre-index period. In our study, ischaemic VT was defined by the presence of MI in the 6-month pre-index period which was consistent with the prior studies. ${ }^{41}$

\section{Study outcomes}

Rates of complications including pulmonary embolism (PE), pericarditis, pericardial tamponade and vascular complications including postop-haemorrhage, postophaemorrhage requiring transfusion, injury to blood vessel, accidental puncture, arteriovenous fistula and injury to retro-peritoneum in the 30-day post-index ablation period (including index admission) were examined. A full list of codes used to identify complications are given in online supplemental file 1 . Rates of inpatient readmission due to recurrent VT, heart failure (HF) and non-VT arrhythmias in the 12-month post-index ablation period were also examined. Factors associated with readmission were determined.

\section{Study covariates}

Covariates included patient demographic characteristics (age, gender, region, location in metropolitan statistical area, insurance type and place of service), procedural characteristics (year of ablation) and patients' clinical characteristics (Charlson comorbidity index (CCI), ${ }^{12}$ $\mathrm{CHA}_{2} \mathrm{DS}_{2}$-VASc score, renal disease, valvular heart disease, acute ischaemic stroke, cardiomyopathy, HF and non-VT arrhythmia (including atrial fibrillation, atrial flutter, paroxysmal supra-VT, non-paroxysmal atrioventricular nodal tachycardia, Wolff-Parkinson-White syndrome), ICD/CRT-D use, prior hospitalisation, number of different AADs, anticoagulants and diuretics used). Patients' clinical characteristics were measured in the 6-month pre-index period.

\section{Statistical analyses}

Descriptive statistics were reported for all study variables. Rate of complications in the 30-day post-index ablation (including index admission) period were reported. Rates of 30-day, 60-day, 90-day and 12-month inpatient readmission following index-ablation were reported.

Cox regression models (multivariable HRs; 95\% CIs were used to evaluate the factors associated with inpatient readmission in the 12-month post-index ablation 


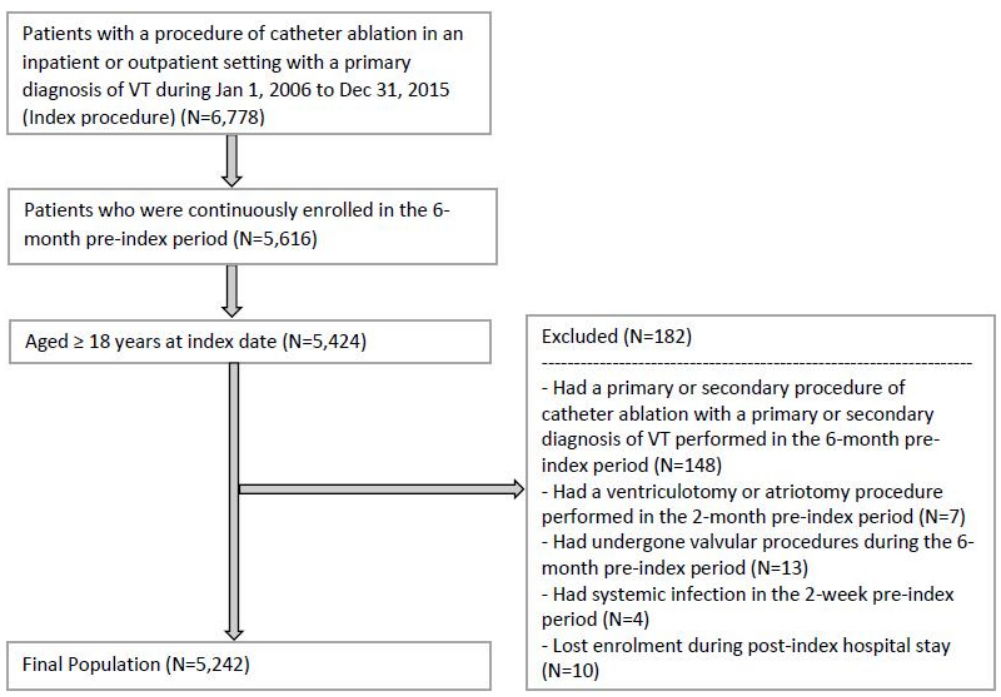

Figure 1 Patient attrition flow diagram. VT, ventricular tachycardia.

period. All analyses were stratified across ischaemic and non-ischaemic VT patients. SAS for Windows, V.9.4 (SAS Institute) was used for study analyses.

\section{RESULTS \\ Patient characteristics}

A final sample of 5242 patients (488 with ischaemic and 4754 with non-ischaemic VT) was identified after applying the inclusion and exclusion criteria (figure 1). Characteristics of the patients included in the analytical cohort are presented in table 1. For patients with ischaemic VT, $82 \%$ were in the age category $50-64,81 \%$ were men, and the majority $(63 \%)$ had prior ICD implantation. However, among patients with non-ischaemic VT, the age-sex distribution was equitably balanced $54 \%$ in the age category $50-64 \%$ and $50 \%$ men) and only $17 \%$ had a baseline ICD implantation. The majority of the ablations occurred in an outpatient setting (57\% for ischaemic and $66 \%$ for non-ischaemic VT).

Roughly $52 \%$ of patients with ischaemic VT and $8 \%$ of patients with non-ischaemic VT had a CCI score of $\geq 3$. Majority (61\%) of patients in the ischaemic VT cohort had a $\mathrm{CHA}_{2} \mathrm{DS}_{2}$-VASc score $\geq 3$, while $18 \%$ of non-ischaemic VT patients had that score. The most common comorbidity in the ischaemic VT cohort was HF $(53 \%)$ followed by cardiomyopathy (50\%). Cardiomyopathy also was the most common comorbidity (22\%) among patients with nonischaemic VT followed by valvular heart disease (21\%). Study characteristics for ischaemic and non-ischaemic VT patients in final sample are presented in table 1.

\section{Rate of complications}

Vascular complications $(2.05 \%$ and $1.6 \%$ for ischaemic and non-ischaemic VT patients, respectively) were the most common complications among the four different complications studied, followed by pericardial tamponade $(1.23 \%$ and $1.37 \%$ for ischaemic and non-ischaemic VT patients, respectively) and $\mathrm{PE}(1.23 \%$ and $0.55 \%$ for ischaemic and non-ischaemic VT patients, respectively) in the 30-day post-index ablation period (including index admission). Pericarditis occurrence rate was the lowest with $0.41 \%$ for ischaemic VT patients and $0.36 \%$ for nonischaemic VT patients in the 30-day post-index ablation period (tables 2 and 3 ).

\section{Rate and predictors of inpatient readmissions}

Among ischaemic VT patients, $17 \%, 7.6 \%$ and $4.7 \%$ had VT-related, HF-related and non-VT arrhythmia-related inpatient readmissions, respectively in the 12-month postindex ablation period (table 2). Among non-ischaemic VT patients, $7.5 \%, 1.7 \%$ and $3.1 \%$ had VT, HF and non-VT arrhythmia-related inpatient readmissions, respectively in the 12-month post-index ablation period (table 3 ).

Results from the regression analyses revealed inpatient setting (vs outpatient) of index ablation (HR 1.82; 95\% CI 1.10 to 3.04), baseline ICD/CRT-D implantation (HR 2.81; $95 \%$ CI 1.36 to 5.79 ), HF (HR 2.66; $95 \%$ CI 1.37 to 5.18 ) and $\geq 2$ prior hospitalisation (HR 2.24; 95\% CI 1.11 to $4.51)$ to be associated with a higher risk of post-ablation 12-month VT-related inpatient readmission among patients with ischaemic VT. Inpatient setting (vs outpatient) of index ablation (HR 2.75; 95\% CI 1.20 to 6.29) and baseline HF comorbidity (HR 8.11; 95\% CI 2.27 to 29.02) also were significant predictors of HF-related readmission among these patients. Additionally, patients with baseline renal disease compared with those without a renal disease (HR 3.35 ; $95 \%$ CI 1.29 to 8.72 ) were about three times more likely to be readmitted to hospital for HF-related causes following VT ablation. Prior use of at least two diuretics (vs 0: HR $6.03 ; 95 \%$ CI 1.43 to 25.45 ) was the only factor found to be significantly associated with a higher risk of postablation non-VT arrhythmia related inpatient readmission (figure 2).

Similar factors also emerged significant with VT-related inpatient readmission among non-ischaemic VT patients (figure 3). Prior hospitalisation ( $\geq 2$ ) was a significant 


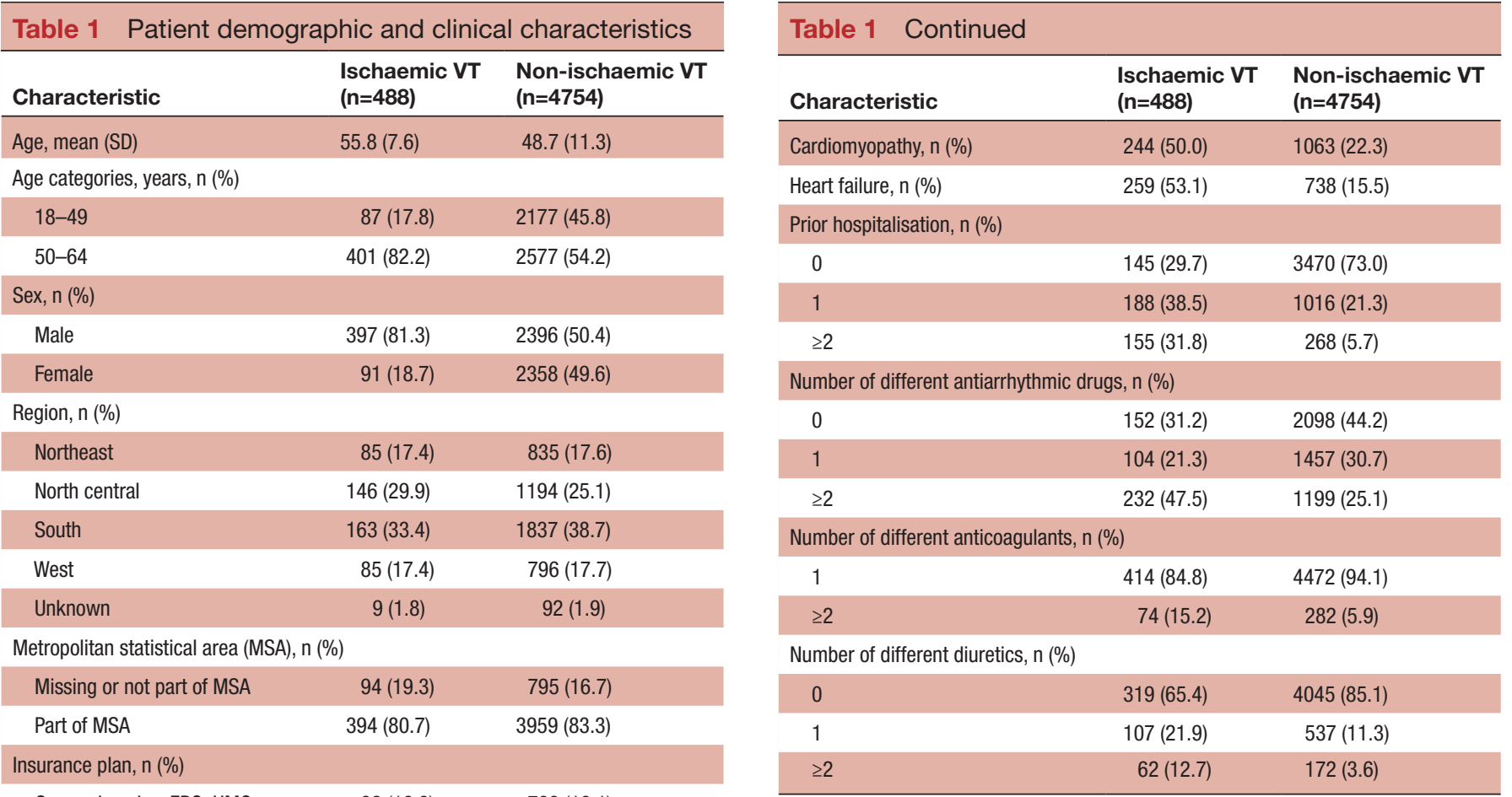

\begin{tabular}{|c|c|c|}
\hline Comprehensive, EPO, HMO & $82(16.8)$ & $768(16.1)$ \\
\hline POS, POS with Capitation, PPO & $338(69.3)$ & $3408(71.7)$ \\
\hline CDHP, HDHP & $30(6.1)$ & $342(7.2)$ \\
\hline Missing & $38(7.8)$ & $236(5.0)$ \\
\hline \multicolumn{3}{|l|}{ Place of service, $n$ (\%) } \\
\hline Inpatient & $209(42.8)$ & $1600(33.6)$ \\
\hline Outpatient & $279(57.2)$ & $3154(66.4)$ \\
\hline ICD/CRT-D use, n (\%) & $307(62.9)$ & $821(17.2)$ \\
\hline \multicolumn{3}{|l|}{ Index year, n (\%) } \\
\hline 2006 & $15(3.1)$ & $283(5.9)$ \\
\hline 2007 & $27(5.5)$ & $325(6.8)$ \\
\hline 2008 & $30(6.2)$ & $343(7.2)$ \\
\hline 2009 & $50(10.3)$ & $429(9.1)$ \\
\hline 2010 & $37(7.6)$ & $486(10.2)$ \\
\hline 2011 & $77(15.8)$ & $610(12.9)$ \\
\hline 2012 & $64(13.1)$ & $757(15.9)$ \\
\hline 2013 & $68(13.9)$ & $594(12.5)$ \\
\hline 2014 & $70(14.3)$ & $581(12.3)$ \\
\hline 2015 & $50(10.3)$ & $346(7.3)$ \\
\hline \multicolumn{3}{|l|}{ Charlson comorbidity index, $\mathrm{n}(\%)$} \\
\hline 0 & 0 & $2573(54.2)$ \\
\hline $1-2$ & $233(47.8)$ & $1795(37.7)$ \\
\hline$\geq 3$ & $255(52.3)$ & $386(8.1)$ \\
\hline \multicolumn{3}{|l|}{$\mathrm{CHA}_{2} \mathrm{DS}_{2}$-VASc score, $\mathrm{n}(\%)$} \\
\hline 0 & 0 & $837(17.6)$ \\
\hline $1-2$ & $190(38.9)$ & $3073(64.6)$ \\
\hline$\geq 3$ & $298(61.1)$ & $844(17.8)$ \\
\hline Non-VT arrhythmia, n (\%) & $461(94.5)$ & $4563(96.0)$ \\
\hline Renal disease, $\mathrm{n}(\%)$ & $91(18.7)$ & $170(3.6)$ \\
\hline Valvular heart disease, $\mathrm{n}(\%)$ & $170(34.8)$ & $998(21.0)$ \\
\hline Acute ischaemic stroke, $\mathrm{n}(\%)$ & $37(7.6)$ & $127(2.7)$ \\
\hline
\end{tabular}

All covariates and medication use were measured in the 6-month pre-index period.

CDHP, consumer-driven health plans; EPO, Exclusive Provider Organization; $\mathrm{HDHP}$, high deductible health plan; HMO, Health Maintenance Organization; POS, Point of Service; PPO, Preferred Provider Organization.

predictor of inpatient readmission across VT- (HR 1.98; 95\% CI 1.40 to 2.81), HF-related (HR 0.42; 95\% CI 0.19 to 0.93 ) and non-VT arrhythmia-related (HR $1.95 ; 95 \%$ CI 1.08 to 3.52 ) inpatient readmission among non-ischaemic VT patients. Inpatient setting of index ablation as opposed to outpatient setting was associated with a higher risk of VT-related (HR 1.54; 95\% CI 1.24 to 1.93 ) and non-VT arrhythmia-related (HR 1.75 ; $95 \%$ CI 1.24 to 2.47 ) inpatient readmission.

CCI score ( $\geq 3$ vs 0: HR 3.86; 95\% CI 1.93 to 7.72 ) was a significant predictor only for HF related inpatient readmission among non-ischaemic VT patients. $\mathrm{CHA}_{2} \mathrm{DS}_{2}-\mathrm{VASc}$ score was not found to be a significant predictor of inpatient readmission in either ischaemic VT patients $(\geq 3 \mathrm{vs}$ 1-2-VT-related: HR 0.55; 95\% CI 0.28 to 1.10 ; HF-related: HR 0.57 ; $95 \%$ CI 0.17 to 1.90 ; and non-VT arrhythmiarelated: HR $0.52 ; 95 \% \mathrm{CI} 0.14$ to 0.92$)$ or non-ischaemic VT patients ( $\geq 3$ vs 0 -VT-related: HR $0.87 ; 95 \%$ CI 0.55 to 1.40; HF-related: HR 2.03; $95 \%$ CI 0.41 to 10.14 ; and non-VT arrhythmia-related: HR $0.79 ; 95 \%$ CI 0.39 to 1.60 ). Full detail of factors affecting inpatient readmission is depicted in figure 2 (ischaemic VT patients) and figure 3 (non-ischaemic VT patients).

\section{DISCUSSION}

To the best of our knowledge, this is one of the first studies to examine outcomes among both ischaemic and nonischaemic VT patients treated with catheter ablation in a real-world setting. In our study, ischaemic VT was defined 


\begin{tabular}{|c|c|c|c|c|}
\hline Outcomes & 30-day, n (\%)* & 60-day, n (\%)* & 90-day, n (\%)* & 12-month, n (\%)* \\
\hline VT readmission† & $31(6.35)$ & $49(10.04)$ & $56(11.48)$ & 83 (17.01) \\
\hline HF readmission $\dagger$ & $10(2.05)$ & $14(2.87)$ & $17(3.48)$ & $37(7.58)$ \\
\hline Non-VT arrhythmia readmission† & $5(1.02)$ & $7(1.43)$ & $10(2.05)$ & $23(4.7)$ \\
\hline \multicolumn{5}{|l|}{ Complications } \\
\hline Totalł & $20(4.10)$ & NA & NA & NA \\
\hline Pulmonary embolism $\ddagger$ & $6(1.23)$ & NA & NA & NA \\
\hline Pericarditis $\ddagger$ & $2(0.41)$ & NA & NA & NA \\
\hline Pericardial tamponade $\ddagger$ & $6(1.23)$ & NA & NA & NA \\
\hline Vascular complicationsł & $10(2.05)$ & NA & NA & NA \\
\hline
\end{tabular}

The total complication represents either of the four complications studied in the post-index period (including index admission). Each patient might have multiple complications and thus the total complications do not add up to the individual number of complications.

*Per cent calculated as of the total 488 patients with ischaemic VT.

†12-month post index-ablation period.

$\$ 30$-day post-index ablation (including index admission) period.

$\mathrm{HF}$, heart failure; VT, ventricular tachycardia.

by the presence of MI in the 6-month pre-index period. Palaniswamy et at and Cheung et $a l^{11}$ also identified patients with ischaemic VT based on a prior history of MI from Nationwide Inpatient Sample (NIS) database and Nationwide Readmissions Database, respectively. Our definition of ischaemic VT was consistent with the prior studies in order to better compare the results with these studies.

Though a few studies have reported outcomes for ischaemic VT patients, such information for non-ischaemic VT patients treated with catheter ablation has been lacking in the literature. Newer evidence suggests that there is an increase in catheter ablation for non-ischaemic VT. ${ }^{13-15}$ Briceño and colleagues using the NIS database reported that from 2003 to 2014, 133529 patients were hospitalised for non-ischaemic VT out of which 14651 underwent catheter ablation. ${ }^{13}$ Catheter ablation rate increased from $9.3 \%$ to $12.1 \%$ during this period. ${ }^{13}$ Della
Bella and Trevisi also reported that non-ischaemic VT ablations accounted for more than $50 \%$ of all ablations in many tertiary ablation centres. ${ }^{16}$ The fact that nonischaemic VT patients constituted a larger sample than ischaemic VT patients in our study further highlights the need to better understand outcomes in this population.

In terms of complications, vascular complications, due to vascular access site, were the most commonly reported complications among both ischaemic $(2.05 \%)$ and nonischaemic VT (1.6\%) patients in the 30-day post-index period, consistent with results of prior VT ablation studies. ${ }^{41718}$ In their single centre study assessing outcomes among patients undergoing VT ablation, Peichl et $a l^{17}$ reported a 30-day vascular complication rate of $4.7 \%$ and $1.6 \%$ for structural heart disease-VT and idiopathic VT patients, respectively. Palaniswamy et at ${ }^{4}$ also found vascular complication to be the most common complication among

\begin{tabular}{|c|c|c|c|c|}
\hline Outcomes & 30-day, n (\%)* & 60-day, n (\%)* & 90-day, n (\%)* & 12-month, n (\%)* \\
\hline VT readmission† & $134(2.82)$ & $194(4.08)$ & $230(4.83)$ & 356 (7.49) \\
\hline HF readmission† & $20(0.42)$ & $28(0.59)$ & $34(0.72)$ & $79(1.66)$ \\
\hline Non-VT arrhythmia readmission† & $37(0.78)$ & $58(1.22)$ & $73(1.54)$ & $147(3.09)$ \\
\hline \multicolumn{5}{|l|}{ Complications } \\
\hline Totalł & $145(3.05)$ & NA & NA & NA \\
\hline Pulmonary embolism $\ddagger$ & $26(0.55)$ & NA & NA & NA \\
\hline Pericarditisł & $17(0.36)$ & NA & NA & NA \\
\hline Pericardial tamponadeł & $65(1.37)$ & NA & NA & NA \\
\hline Vascular complicationsł & $76(1.60)$ & NA & NA & NA \\
\hline
\end{tabular}

The total complication represents either of the four complications studied in the post-index period (including index admission). Each patient might have multiple complications and thus the total complications do not add up to the individual number of complications.

*Per cent calculated as of the total 4754 patients with non-ischaemic VT.

†12-month post index-ablation period.

$\ddagger 30$-day post-index ablation (including index admission) period.

$\mathrm{HF}$, heart failure; VT, ventricular tachycardia. 


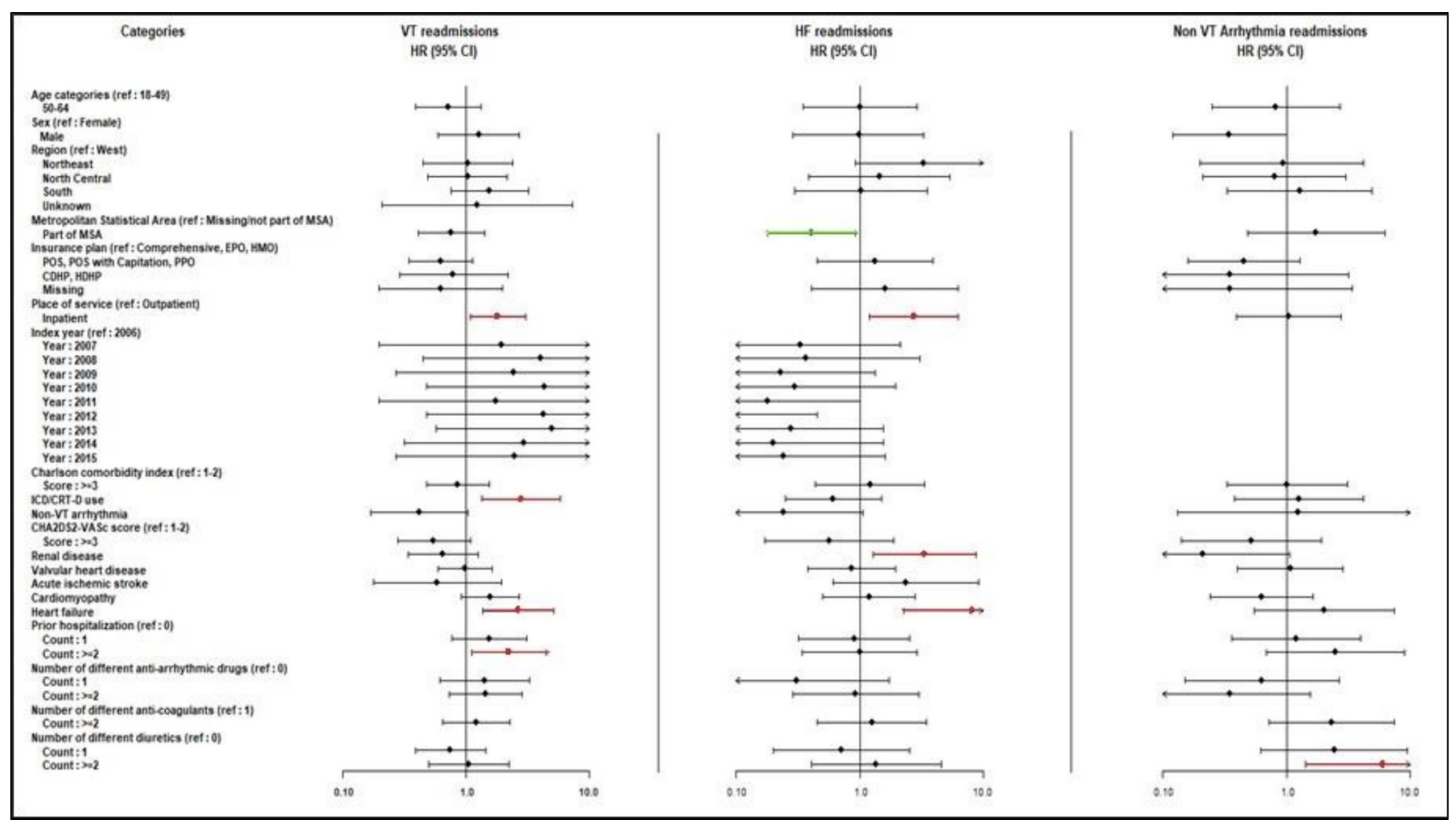

Figure 2 Predictors of inpatient readmission among patients with ischaemic VT in the 12-month post-index ablation period. Factors that significantly increased or decreased the risk of readmission were marked with red or green, respectively. Black lines represent insignificant predictors. All covariates and medication use were measured in the 6-month pre-index period. Missing HRs for the year variable for non-VT arrhythmia related readmission represent very few readmission counts over the years to be reported in the multivariable analysis. CDHP, consumer-driven health plans; EPO, Exclusive Provider Organization; HDHP, high deductible health plan; HMO, Health Maintenance Organization; MSA, metropolitan statistical area; POS, Point of Service; PPO, Preferred Provider Organization; VT, ventricular tachycardia.

patients with ischaemic VT undergoing ablation, with a rate of $6.9 \%$ (in-hospital). Though we could not compare statistically, our rate of vascular complication seems to be at the lower end of those reported in these prior studies, which could be because of multiple factors. First, our study included a relatively healthy sample of commercially insured patients undergoing VT ablation. The average age of our sample was around 56 years for ischaemic VT patients and 49 years for non-ischaemic VT patients. In contrast, the Peichl et alstudy had an average age of 62 years for ischaemic VT patients and 50 years for non-ischaemic VT patients. ${ }^{17}$ The average age of ischaemic VT patients undergoing ablation in the Palaniswamy et at $t^{t}$ study was 66 years. Second, we included patients who had VT ablation in either the inpatient or outpatient setting, with most of our sample (57\% for ischaemic and $66 \%$ for non-ischaemic VT patients) having ablation in an outpatient setting. In contrast, Palaniswamy et al assessed complications in ischaemic VT patients who had ablation performed only in an inpatient setting. Differential case-mix between the two studies may likely be a cause for differences in outcomes. Finally, our data represented more recent years, and potentially captured patients who underwent ablation using ablation catheters with advanced features like contact force and porous tip technology along with more emphasis on increasing safety for vascular access by using ultrasound guidance and other protocols being adopted across institutions in the country. Irrigated radiofrequency (RF) technology has shown long-term positive outcomes in terms of prevention of recurrent VT and complications. ${ }^{31920}$ The data period for Peichl et al went until 2012 and for Palaniswamy et al until 2011, which represent a time frame when these advanced catheters were not available. Further research is needed to better understand the change in complications rate over the years especially with recent advancements in RF catheter technology.

Among patients with ischaemic VT, about $17 \%$ of patients had a VT-related readmission in the 12-month post-index ablation period. We observed a lower rate of HF (7.6\%) and non-VT arrhythmia (4.7\%) related inpatient readmission among these patients. Yousuf and colleagues found a VT-related inpatient readmission rate of 18 per 100 person-years and HF-related rate of 15.4 per 100 person-years in the 1-year period following catheter ablation among Medicare population. ${ }^{18}$ Cheung et $a l^{11}$ found a 30-day VT- and HF-related readmissions of 7.9\% and $2.8 \%$, respectively among ischaemic VT patients undergoing ablation. We observed a readmission rate of about $6 \%$ or less in the 30 days following ablation among ischaemic VT patients. As with reasons discussed for the findings associated with lower complication rate in our study, some of those factors including age differential and case-mix studied could have attributed to the lower rate of readmission observed in our study. Marchlinski 


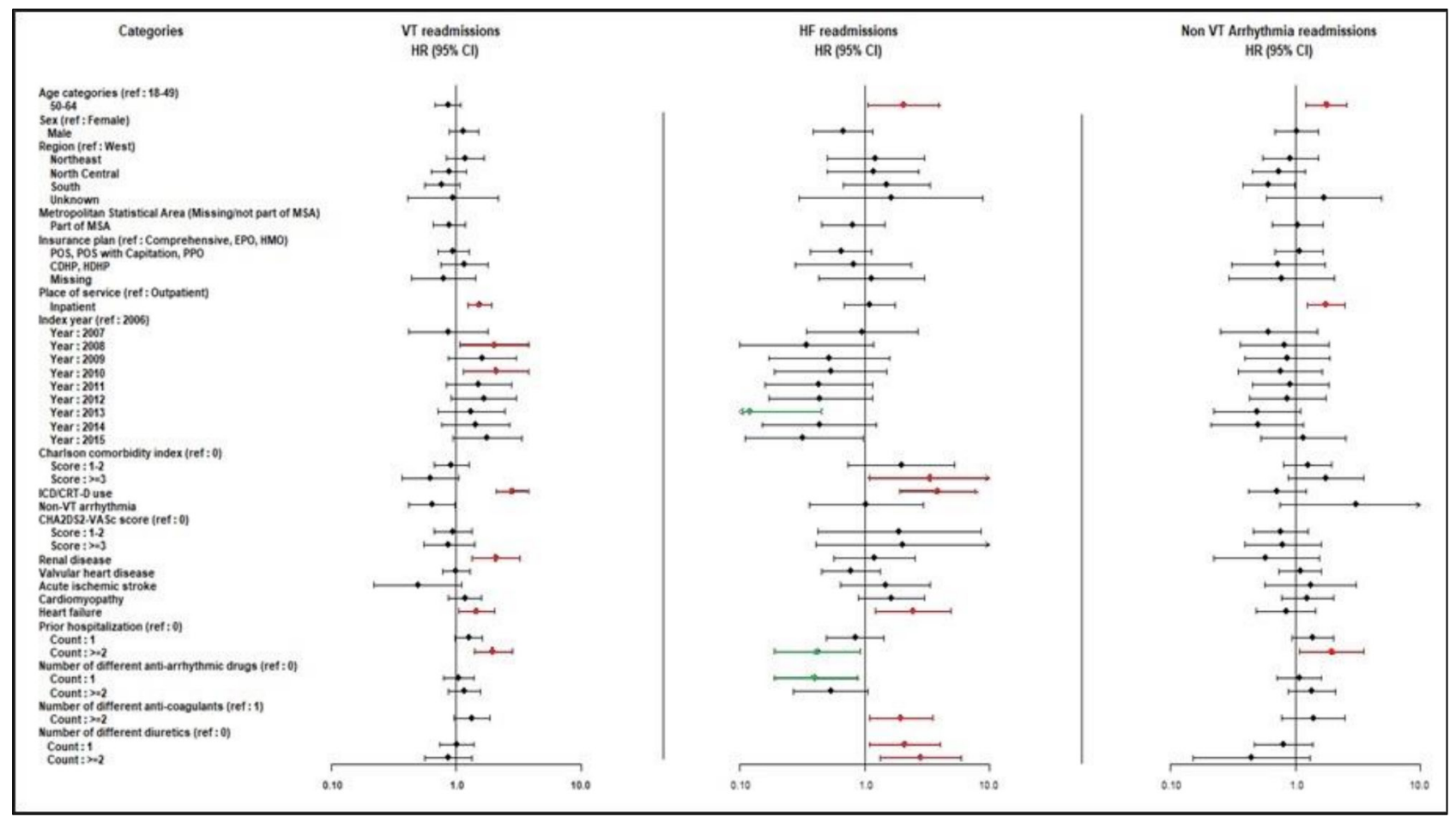

Figure 3 Predictors of inpatient readmission among patients with non-ischaemic VT in the 12-month post-index ablation period. Factors that significantly increased or decreased the risk of readmission were marked with red or green, respectively. Black lines represent insignificant predictors. All covariates and medication use were measured in the 6-month pre-index period. CDHP, consumer-driven health plans; EPO, Exclusive Provider Organization; HDHP, high deductible health plan; HMO, Health Maintenance Organization; MSA, metropolitan statistical area; POS, Point of Service; PPO, Preferred Provider Organization; VT, ventricular tachycardia.

and colleagues found a reduction in ICD shocks and VT episodes and improved quality of life at 6 months following catheter ablation..$^{20}$ The authors also found a decrease in amiodarone use and hospitalisation in the 3 -year period. ${ }^{20}$ In our study, among patients with nonischaemic VT, the 30-day readmission rates were even lower with only $2.8 \%, 0.4 \%$ and $0.8 \%$ readmissions for VT, HF and non-VT arrhythmia related causes, respectively. It is difficult to place these rates into context, given the lack of information on readmissions for nonischaemic VT patients. Such low rates of complications do reflect the favourable safety profile of VT ablation in this population cohort.

Prior hospitalisation and procedural setting were significantly associated with an increased risk of readmission. These outcomes suggest that patient case-mix and prior healthcare utilisation play an influential role in post-ablation resource utilisation. Some individual comorbidities such as HF and renal disease were significantly associated with a higher risk of inpatient readmission which are commensurate with some of the prior studies. ${ }^{11} 18$ Among ischaemic VT patients, for instance, we observed that patients with renal disease were about three times more likely to be readmitted to hospital for HF-related causes following VT ablation. Likewise, Yousuf et $a l^{18}$ found that the presence of renal disease increased the risk of HF-related readmission by twice in the 1-year post ablation follow-up period. In our study, ischaemic
VT patients with baseline HF were more than two times as likely to have VT-related inpatient readmission in the 12-month post-index ablation period compared with patients without HF.

Our study has a few limitations. As is true for most realworld large administrative datasets, the IBM MarketScan Commercial database used for the study lacked clinical granularity, including variables such as LV ejection fraction, New York Heart Association functional class, information on actual ICD shocks and mortality. We also did not have information regarding the hospital ablation volume or provider experience, which could likely affect the study outcomes. In our study, ischaemic VT was defined by the presence of a diagnosis code for MI in the 6-month preindex period. We used these criteria to be consistent with past studies; however, by using this criteria we may be inaccurately reporting the true incidence of ischemic VT. For example, in patients with non-ischemic cardiomyopathy who had type II or small MI in addition to missing cases with possible non-documented MIs in their recent visits, the actual proportion of ischaemic VT patients may not be fully known in the study. Further, patients who did not have any medical services visit where MI was listed in the diagnosis field will be missed as well. Additionally, our reliance on diagnosis and procedure codes to define study variables could be subject to miscoding or misclassification. For example, we observed that $63 \%$ of the patients undergoing ablation for ischaemic VT had prior ICD implants. Ideally, 
it should be close to $100 \%$ and this under-reporting could be a function of miscoding in the claims database. Additionally, we identified the presence of ICD implant in the 6-month pre-index period. It is also possible that patients had ICD implants prior to that which might not have captured here. Finally, this study focused on non-elderly patients using commercial claims database and thus the study findings might not be generalisable to the entire US population.

\section{CONCLUSION}

This study examined complications and readmissions following ablation in a sample of ischaemic and nonischaemic VT patients. Vascular complications were observed to be the most common complication for both ischaemic and non-ischaemic VT patients. The lower rate of complications compared with the prior studies could be attributed to device and/or non-device related factors.

Almost $17 \%$ of ischaemic VT patients and $7 \%$ of nonischaemic VT patients had a VT-related readmission in the 12-month post-index ablation period. Ablation setting and number of past hospitalisations emerged significant predictors of readmissions. As ablation plays an increasingly important role for the management and treatment of VT patients, our results suggest that the procedural risks are relatively low and measures of effectiveness that can be obtained from large administrative claims databases indicate relatively low rates of readmissions following the procedures in this study sample of a younger cohort of patients.

Contributors VM, IK and RK designed the study; RK and MR reviewed the literature; RK, SY and MR analysed the data; VM, LMB, NG, IK, SZ, SY, RK and MR interpreted the study findings; VM, RK and MR wrote the first draft of the manuscript; LMB, NG, IK and SZ contributed to the subsequent drafts and reviewed/edited the manuscript. All authors read and approved the final manuscript.

Funding This work was supported by Johnson \& Johnson.

Competing interests All other authors are employees of the Johnson \& Johnson Family of Companies.

Patient consent for publication Not required.

Ethics approval The study data is based on fully de-identified patient level information and in compliance with the Health Information Portability and Accountability Act of 1996 (HIPAA).

Provenance and peer review Not commissioned; externally peer reviewed.

Data availability statement All data relevant to the study are included in the article or uploaded as supplementary information.

Open access This is an open access article distributed in accordance with the Creative Commons Attribution Non Commercial (CC BY-NC 4.0) license, which permits others to distribute, remix, adapt, build upon this work non-commercially, and license their derivative works on different terms, provided the original work is properly cited, appropriate credit is given, any changes made indicated, and the use is non-commercial. See: http://creativecommons.org/licenses/by-nc/4.0/.

ORCID iD

Motiur Rahman http://orcid.org/0000-0002-3890-3446

\section{REFERENCES}

1 Priori SG, Blomström-Lundqvist C, Mazzanti A, et al. 2015 ESC guidelines for the management of patients with ventricular arrhythmias and the prevention of sudden cardiac death: the task force for the management of patients with ventricular arrhythmias and the prevention of sudden cardiac death of the European Society of cardiology (ESC). endorsed by: association for European paediatric and congenital cardiology (AEPC). Eur Heart J 2015;36:2793-867.

2 Koplan BA, Stevenson WG. Ventricular tachycardia and sudden cardiac death. Mayo Clin Proc 2009;84:289-97.

3 Timothy M, Markman DAM, Liang JJ. Catheter ablation for ventricular tachycardia in patients with structural heart disease. US Cardiol Rev 2018;12:51-6.

4 Palaniswamy C, Kolte D, Harikrishnan P, et al. Catheter ablation of postinfarction ventricular tachycardia: ten-year trends in utilization, in-hospital complications, and in-hospital mortality in the United States. Heart Rhythm 2014;11:2056-63.

5 European Heart Rhythm Association, Heart Rhythm Society, Zipes DP, et al. ACC/AHA/ESC 2006 guidelines for management of patients with ventricular arrhythmias and the prevention of sudden cardiac death: a report of the American College of Cardiology/American heart association Task force and the European Society of cardiology Committee for practice guidelines (writing Committee to develop guidelines for management of patients with ventricular arrhythmias and the prevention of sudden cardiac death). J Am Coll Cardiol 2006;48:e247-346.

6 Stevenson WG, Wilber DJ, Natale A, et al. Irrigated radiofrequency catheter ablation guided by electroanatomic mapping for recurrent ventricular tachycardia after myocardial infarction: the multicenter thermocool ventricular tachycardia ablation trial. Circulation 2008;118:2773-82.

7 Sapp JL, Wells GA, Parkash R, et al. Ventricular tachycardia ablation versus escalation of antiarrhythmic drugs. $N$ Engl J Med 2016;375:111-21.

8 Kuck K-H, Schaumann A, Eckardt L, et al. Catheter ablation of stable ventricular tachycardia before defibrillator implantation in patients with coronary heart disease (VTACH): a multicentre randomised controlled trial. Lancet 2010;375:31-40.

9 Mukherjee RK, O'Neill L, O'Neill MD. Prophylactic catheter ablation for ventricular tachycardia: are we there yet? Arrhythm Electrophysiol Rev 2017;6:125-8.

10 Ladapo JA, David G, Gunnarsson CL, et al. Healthcare utilization and expenditures in patients with atrial fibrillation treated with catheter ablation. J Cardiovasc Electrophysiol 2012;23:1-8.

11 Cheung JW, Yeo I, Ip JE, et al. Outcomes, costs, and 30-day readmissions after catheter ablation of myocardial InfarctAssociated ventricular tachycardia in the real world: nationwide readmissions database 2010 to 2015. Circ Arrhythm Electrophysiol 2018;11:e006754.

12 Quan H, Sundararajan V, Halfon P, et al. Coding algorithms for defining comorbidities in ICD-9-CM and ICD-10 administrative data. Med Care 2005;43:1130-9.

13 Briceño DF, Gupta T, Romero J, et al. Catheter ablation of ventricular tachycardia in nonischemic cardiomyopathy: a propensity scorematched analysis of in-hospital outcomes in the United States. J Cardiovasc Electrophysiol 2018;29:771-9.

14 Zeppenfeld K. Ventricular tachycardia ablation in nonischemic cardiomyopathy. JACC Clin Electrophysiol 2018;4:1123-40.

15 Dinov B, Fiedler L, Schönbauer R, et al. Outcomes in catheter ablation of ventricular tachycardia in dilated nonischemic cardiomyopathy compared with ischemic cardiomyopathy: results from the prospective heart centre of Leipzig VT (HELP-VT) study. Circulation 2014;129:728-36.

16 Della Bella P, Trevisi N. Catheter ablation of ventricular tachycardia in nonischemic dilated cardiomyopathy: a difficult field where we should focus our efforts. Circ Arrhythm Electrophysiol 2016;9.

17 Peichl P, Wichterle D, Pavlu L, et al. Complications of catheter ablation of ventricular tachycardia: a single-center experience. Circ Arrhythm Electrophysiol 2014;7:684-90.

18 Yousuf OK, Zusterzeel R, Sanders W, et al. Trends and outcomes of catheter ablation for ventricular tachycardia in a community cohort. JACC Clin Electrophysiol 2018;4:1189-99.

19 Müssigbrodt A, Grothoff M, Dinov B, et al. Irrigated tip catheters for radiofrequency ablation in ventricular tachycardia. Biomed Res Int 2015;2015:389294.

20 Marchlinski FE, Haffajee $\mathrm{Cl}$, Beshai JF, et al. Long-Term success of irrigated radiofrequency catheter ablation of sustained ventricular tachycardia: Post-Approval THERMOCOOL VT trial. J Am Coll Cardiol 2016;67:674-83. 